\title{
Comparison of coplanar and noncoplanar intensity-modulated radiation therapy and helical tomotherapy for hepatocellular carcinoma
}

\author{
Chen-Hsi Hsieh1,6, Chia-Yuan Liu4, Pei-Wei Shueng 1,7, Ngot-Swan Chong', Chih-Jen Chen', Ming-Jen Chen4, Ching- \\ Chung Lin'4, Tsang-En Wang ${ }^{4}$, Shee-Chan Lin ${ }^{4}$, Hung-Chi Tai ${ }^{3}$, Hui-Ju Tien ${ }^{1}$, Kuo-Hsin Chen², Li-Ying Wang ${ }^{9}$, Yen- \\ Ping Hsieh ${ }^{10}$, David YC Huang ${ }^{* 11}$ and Yu-Jen Chen*3,5,6,8
}

\begin{abstract}
Background: To compare the differences in dose-volume data among coplanar intensity modulated radiotherapy (IMRT), noncoplanar IMRT, and helical tomotherapy (HT) among patients with hepatocellular carcinoma (HCC) and portal vein thrombosis (PVT).

Methods: Nine patients with unresectable HCC and PVT underwent step and shoot coplanar IMRT with intent to deliver 46 - 54 Gy to the tumor and portal vein. The volume of liver received 30Gy was set to keep less than 30\% of whole normal liver (V30<30\%). The mean dose to at least one side of kidney was kept below $23 \mathrm{~Gy}$, and 50 Gy as for stomach. The maximum dose was kept below $47 \mathrm{~Gy}$ for spinal cord. Several parameters including mean hepatic dose, percent volume of normal liver with radiation dose at X Gy $(V x)$, uniformity index, conformal index, and doses to organs at risk were evaluated from the dose-volume histogram.

Results: $\mathrm{HT}$ provided better uniformity for the planning-target volume dose coverage than both IMRT techniques. The noncoplanar IMRT technique reduces the V10 to normal liver with a statistically significant level as compared to HT. The constraints for the liver in the V30 for coplanar IMRT vs. noncoplanar IMRT vs. HT could be reconsidered as $21 \%$ vs. $17 \%$ vs. $17 \%$, respectively. When delivering 50 Gy and $60-66$ Gy to the tumor bed, the constraints of mean dose to the normal liver could be less than 20 Gy and 25 Gy, respectively.
\end{abstract}

Conclusion: Noncoplanar IMRT and HT are potential techniques of radiation therapy for HCC patients with PVT. Constraints for the liver in IMRT and HT could be stricter than for 3DCRT.

\section{Background}

Hepatocellular carcinoma (HCC) is one of the most common malignancies worldwide [1] and is the third most common cause of cancer mortality in the recent year [2]. The 5-year survival rate of individuals with liver cancer reported by the American Cancer Society in the United States is less than $10 \%$ despite aggressive conventional therapy. In addition, comparing 1991 and 2005, liver cancer is not only one of the three cancers with an increasing death rate, but also the fastest growing death rate $(27 \%)$ in

\footnotetext{
*Correspondence: huangd@mskcc.org, chenmdphd@yahoo.com

3 Department of Radiation Oncology, Mackay Memorial Hospital, Taipei, Taiwan

11 Department of Medical Physics, Memorial Sloan-Kettering Cancer Center, New York, NY, USA

Full list of author information is available at the end of the article
}

the United States [3]. Portal vein thrombosis (PVT) is a common complication in patients with advanced-stage HCC, occurring in $20 \%-80 \%$ of these patients [4-6]. PVT may alter the correct evaluation of HCC imaging and also limits HCC treatment choices [7]. The median survival time of HCC patients with PVT is approximately 0.7 to 1.6 months without any treatment [8]. Furthermore, PVT is often a poor prognostic factor for patient survival $[9,10]$.

Several modalities, including surgical resection [11], transcatheter arterial chemoembolization (TACE) [12] and arterial infusion chemotherapy [13], percutaneous ethanol injection therapy, microwave coagulation therapy, radiotherapy, and liver transplantation, have been used in treating HCC [14]. However, there are some limi- 
tations to performing these treatments. For example, surgical treatment can only be performed on highly selected patients, because there is a potential risk of postoperative liver failure and early disease recurrence. TACE is considered a contraindication for HCC patients with main portal trunk obstruction and indwelling catheters or catheter-related sepsis, which hinder arterial infusion chemotherapy.

While the role of radiotherapy was limited in the past because of poor tolerance of the whole liver to radiation [15], some studies show that higher irradiation doses resulted in a higher survival rates for HCC patients [16]. Kim DY et al. reported a dose-response relationship exists between the radiation dose and PVT, where the objective response of PVT was observed in 3 of 15 patients (20\%) with BED $<58 \mathrm{~Gy}_{10}$ and in 24 of 44 patients (54.6\%) with BED $\geqq 58 \mathrm{~Gy}_{10}$ [17]. Toya $\mathrm{R}$ et al. [18] pointed out conformal radiotherapy is effective not only for tumor response but also for survival of HCC patients with PVT. We also reported one HCC patient with PVT who received intensity-modulated radiation therapy (IMRT) with sorafenib, resulting in a significant response and improvement [19]. Moreover, radiotherapy could be an effective treatment choice for selected HCC patients with PVT [20].

With advances in radiotherapy modalities, such as three-dimensional conformal radiotherapy (3DCRT) and IMRT, delivering a good radiation dose to the tumor target volume while sparing the critical organs appears achievable $[19,21,22]$. With the development of conformal assays, radiation therapy could be an effective choice for selected HCC patients with PVT [20]. Rotational IMRT modalities, including helical tomotherapy $(\mathrm{HT})$ [23], VMAT (Volumetric intensity modulated arc therapy) [19] and the others, are new image-guided intensitymodulated radiotherapy. These complex rotational IMRT machines can deliver highly conformal dose distributions and possess the ability to spare critical organs in a greater extent $[19,24]$. We evaluated various radiation plans for HT and IMRT as they are currently used at our department. Due to IMRT can preserve acceptable target coverage and better spare nonhepatic organs among HCC patients than 3DCRT [25]. Therefore, we selected different IMRT planning strategies rather than 3DCRT to compare to HT in our study.

The purpose of this study was to define the potential impact of HT and to compare the differences in dosimetric indicators among coplanar and noncoplanar IMRT and HT among HCC patients with PVT previously documented to have at least partial responses to recannularization and to have undergone repeated TACE after IMRT.

\section{Methods}

\section{Patients}

A retrospective study was performed for nine patients with unresectable HCC and PVT underwent step and shoot coplanar IMRT to treat the tumor and portal vein between January 2007 and June 2007, eight of them were men. Patients with at least partial response to RT, documented by identifiable recannularization using $\mathrm{CT}$ imaging or abdominal ultrasound, and could be subjected to receive repeated TACE after RT were retrospectively enrolled. All patients had stage IIIA HCC (American Joint Committee on Cancer Staging, $6^{\text {th }}$ edition), chronic hepatitis B carriers and underwent TACE before and after IMRT, with an interval of at least 30 days between the two modalities.

\section{Radiation therapy \\ (a) Planning CT and Volume definitions}

All patients were immobilized using Alpha Cradle (Smithers Medical Products, Inc. North Canton, OH, USA) in supine position with arms elevated above head to provide a fixed position during CT scan and radiation therapy. Two series of axial CT images, with and without contrast enhancement, with 5-mm contagious slice thickness including whole liver and kidneys were acquired for each patient. Targets were delineated on non-contrast images under the aids by contrast ones. Treatment planning was performed by using non-contrast images. All patients were treated using coplanar static IMRT. No respiratory control or abdomen compression was applied during the treatment, and the organ motion was taken into account in planning-target volume (PTV). Gross tumor volume (GTV) was defined as the hepatic tumor volume plus PVT visualized by contrasted CT images. GTV was expanded by $0.5 \mathrm{~cm}$ to create clinical target volume (CTV). A non-uniform three dimensional (3D) margin, $0.5 \mathrm{~cm}$ radically and $1.5 \mathrm{~cm}$ cranial-caudally was applied to CTV for creating PTV. The normal liver volume was defined as the total liver volume minus the GTV. (b) Dose prescription and planning objectives

The prescription dose was 44.8 to 54.0 Gy depended on the ratio of PTV volume and nonirradiated liver volume [26]. When nonirradiated liver volume was $<1 / 3,1 / 3-1 / 2$ or $>1 / 2$ of liver volume, the delivered dose could be 40 , 44.8-50.4 or 50-66 Gy, respectively. No patient was given radiation to the entire liver. Treatment was delivered once daily with $1.6-1.8 \mathrm{~Gy}, 5$ fractions per week by a $6-\mathrm{MV}$ linear accelerator (Varian 2100IX, Varian Medical Systems, Palo Alto, CA, USA).

For planning objectives, the mean hepatic dose and dose to $30 \%$ volume of liver was kept less than 30 Gy (V30 $<30 \%)[18,27-29]$. Given that HT is a rotational treatment, volumes of low-dose distributed regions for OARs were generally greater [30]. Thus, volume of normal liver 
received 10 and $20 \mathrm{~Gy}$ (V10, V20) were also investigated for a comparison. For OARs, mean dose to stomach, spleen, kidneys and maximum dose to spinal cord were assessed. The maximum doses were specified as maximum dose to $1 \%$ volume, denoted as D1\% [31]. According to TD5/5 (the tolerance dose leading to 5\% complication rates at 5 years), the mean dose to at least one side of kidney was kept below $23 \mathrm{~Gy}$, and $50 \mathrm{~Gy}$ as for stomach [27]. The maximum dose was kept below $47 \mathrm{~Gy}$ for spinal cord [27].

\section{(c) Description of IMRT and helical tomotherapy techniques}

All targets and OARs were delineated on Eclipse V7.3.10 planning system (Varian Medical System, Palo Alto, CA, USA) and then transferred to Helical Hi-Art Tomotherapy (Tomotherapy, Inc., Madison, Wisconsin, USA) via Digital Imaging and Communications in Medicine (DICOM) protocol. The dose by IMRT was calculated using the Eclipse system. In Eclipse plans, 5-field gantry arrangement for coplanar and noncoplanar static step and shoot IMRT was designed in all cases. Minimum monitor units (MU) for each segment was set to 5 with no more than 40 segments were allowed for each plan. For HT plans, the field width, pitch, and modulation factor $[32,33]$ used for the treatment planning optimization were $2.5 \mathrm{~cm}, 0.32$ and 3.5 , respectively. The dose constraints and the penalties were adjusted accordingly during the optimization process. The dose calculation matrix resolution was $3.0 \mathrm{~mm}$ for Eclipse system and $4.0 \mathrm{~mm}$ for HT. The inverse planning systems performed iterations during optimization process, which were multi-resolution dose calculation for Eclipse-IMRT but algebraic iteration for HT. For final dose calculation, HT employed convolution/superposition algorithms and Eclipse employed Analytical Anisotropic Algorithm.

\section{(d) Conformity index (Cl) and Uniformity index (UI)}

The dose to PTV has been estimated by DVH after normalization. Dose conformity and homogeneity to the PTV and OARs represent the ability to fulfill dose-volume histogram objectives. The conformity index $(\mathrm{CI})$ was originally proposed by Paddick [34] to evaluate the tightness of fit of the planning target volume to the prescription isodose volume in treatment plans as follows,

$$
\mathrm{CI}=\left(V_{P T V} / T V_{P V}\right) /\left(T V_{P V} / V_{T V}\right)
$$

where $\mathrm{V}_{P T V}$ is the volume of PTV, $\mathrm{V}_{T V}$ is the treated volume enclosed by the prescriptiond isodose surface, and $T V_{P V}$ is the portion of the PTV within the prescribed isodose volume. The CI approximates unity means lesser dose to normal tissues and higher dose to target volume. The uniformity index (UI) was defined as $D_{5 \%} / D_{95 \%}$, where $\mathrm{D}_{5 \%}$ and $\mathrm{D}_{95 \%}$ were the minimum doses delivered to $5 \%$ and $95 \%$ of the planning target volume as previously reported [35]. The greater $\mathrm{HI}$ indicates the poorer inhomogeneity.

\section{Statistical methods}

Differences in actuarial outcomes between the three groups were calculated using one-way ANOVA with post hoc multiples comparisons. The differences were considered significant at $\mathrm{p}<0.05$. All analyses were performed using the Statistical Package for the Social Sciences, version 12.0 (SPSS, Chicago, IL, USA).

\section{Results}

Target Volume Coverage, Conformity and Uniformity Index The average CTV and normal liver volume for the nine patients was $614.4 \pm 323.4 \mathrm{ml}$ (range, $154.5-1170.9 \mathrm{ml}$ ) and $1294.8 \pm 372.9 \mathrm{ml}$ (range, 895.4-2125.8 ml), respectively. The isodose distributions in axial, sagittal and coronal views obtained with coplanar IMRT, noncoplanar IMRT and HT in one representative patient were shown in Fig. 1. Fig. 2 shows dose volume histograms (DVHs) for the PTV of one representative patient using coplanar, noncoplanar IMRT and HT planning techniques. In general, the PTV coverage and comformity was better in HT plan. The similar results were obtained for other patients.

For target coverage, $95 \%$ of CTV, $90 \%$ and $95 \%$ of PTV, all achieved at least $99 \%$ of the prescribed dose were listed, respectively. There were no significant differences of coverage for CTV and PTV between three different techniques. (Table 1) The mean score of CI showed no significant difference between the HT and IMRT planning. However, a better uniformity index provided by HT than both IMRT plans was noted $(p<0.05)$ (Table 1$)$. The $\mathrm{UI}$ and $\mathrm{CI}$ for each individual patient were plotted in Fig. 3 and in Fig. 4, respectively.

\section{OARs sparing}

The radiation doses for OARs obtained by coplanar IMRT, noncoplanar IMRT and HT were summarized in Table 1. There were no significant differences between both IMRT techniques and HT for the mean doses of liver. The low dose region of liver for HT plans were higher for V10 than others ( $p$ value $<0.05)$. There was a trend for noncoplanar IMRT and HT that both techniques provided lower V20 and V30 than coplanar IMRT. For other OARs, there were no significant differences between both IMRT and HT plan for spinal cord, kidneys and stomach (Table 1).

\section{Discussion}

Compared with both IMRT techniques, tomotherapy provides better uniformity. The noncoplanar IMRT technique reduced the normal liver volume receiving $10 \mathrm{~Gy}$ to a statistically significant level as compared to tomotherapy. The constraints for V30 of the liver for coplanar 

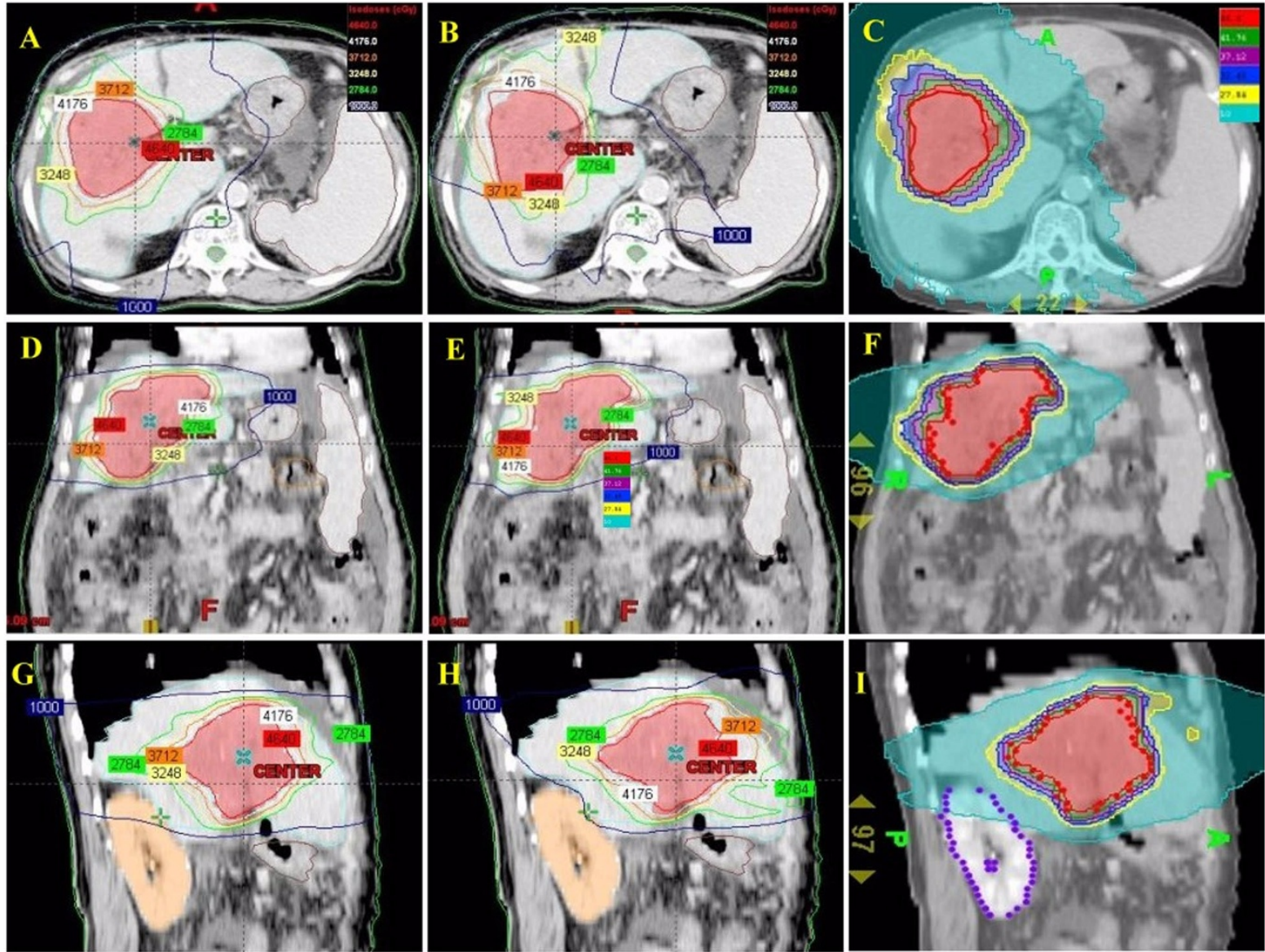

Figure 1 Isodose distributions of prescribed dose of $\mathbf{4 6 . 4}$ Gy to PTV for different treatment techniques. A, D and G showed the isodose axial, coronal and sagittal views for coplanar IMRT plan respectively. B, E and $\mathrm{H}$ were the isodose axial, coronal and sagittal views for noncoplanar IMRT plan. The helical tomotherapy plan was shown in C, F and I.

IMRT $v s$. noncoplanar IMRT $v s$. tomotherapy might be reconsidered as $21 \%$ vs. $17 \%$ vs. $17 \%$, respectively.

Radiotherapy for treating HCC patients has been limited to palliation purpose in the past experiences due to the low tolerance of the whole liver to radiotherapy $[36,37]$ despite HCC being reported as a radiosensitive cancer in clinical investigations [38]. Nevertheless, the radiation dose is the most significant factor associated with tumor response for HCC patients. Troublesomely, as the irradiation doses deliver to the liver increased, hepatic toxicity has become a problem [39]. The encouraging results confirm 3DCRT is an effective modality, not only for tumor response but also for survival in HCC patients who are not suitable for other treatment modalities $[17,18,20]$. Cheng et al. [25] reported that IMRT offers the better potential of increasing the dose conformality to the tumor and reducing the dose to the sensitive structures than 3DCRT does for HCC patients with PVT. Therefore, highly conformality delivered by radiotherapy to HCC patients with PVT cause better tumor control and lower toxicities to normal liver. In the current study, noncoplanar IMRT and HT are compatible with coplanar IMRT in V95 of CTV and PTV. (Table 1) There are no significant differences of CI between HT and both IMRT techniques. However, a trend for more stable conformality for each individual patient provided by HT than both IMRT is noted in Fig. 4. HT provides better uniformity than both IMRT techniques. (Table 1) The UI for each individual patient were plotted in Fig. 3. In addition, the dose-volume histogram for HT had a steeper slope. (Fig. 2) Where the differences among the treatment techniques are clear: suggesting that HT provides higher uniformity within the planning target volume than both IMRT techniques. In summary, HT provides better uniformity for PTV coverage than both IMRT techniques. There is a trend for more stable conformality for each individual patient provided by HT than both IMRT. Nevertheless, both IMRT techniques and HT have similar coverage for V95 of CTV and PTV. 


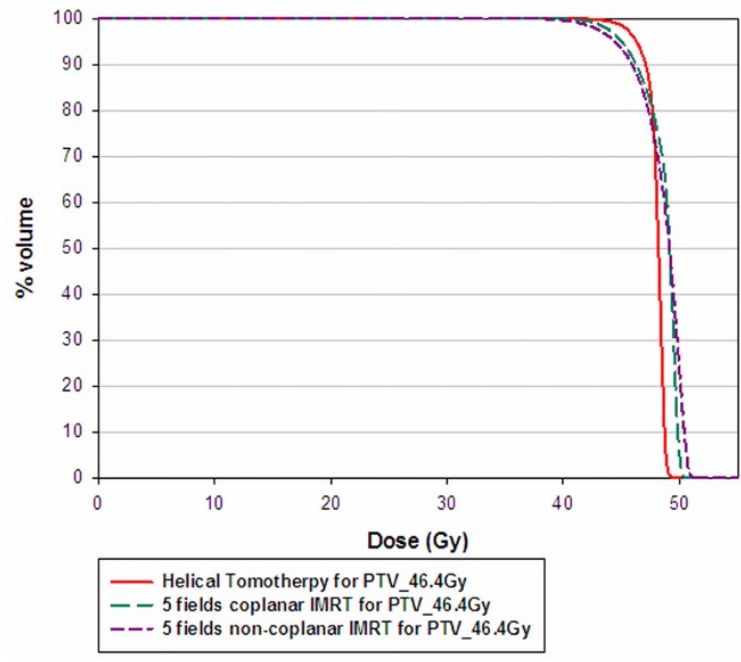

Figure 2 Dose-volume histogram of planning-target volume for one representative patient undergoing coplanar intensity-modulated radiotherapy (IMRT), noncoplanar IMRT, and helical tomotherapy.

There are several models used to predict liver tolerance, one is the normal tissue complication probability (NTCP) and another one is maximum tolerable dose (MTD). The NTCP model shows that the mean liver dose is the most significant predictor of RILD, with a threshold dose of 31 Gy. The University of Michigan Medical Center reported that the mean hepatic dose was a strong predictor of subsequent radiation-induced liver disease (RILD) and no cases of RILD were observed when the

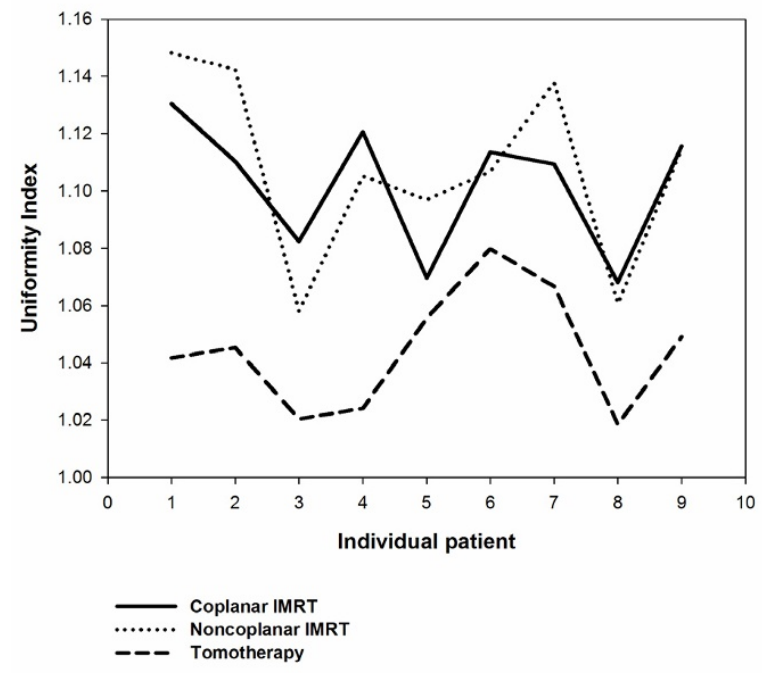

Figure 3 The uniformity index (UI) for each individual patient undergoing coplanar intensity-modulated radiotherapy (IMRT), noncoplanar IMRT, and helical tomotherapy.

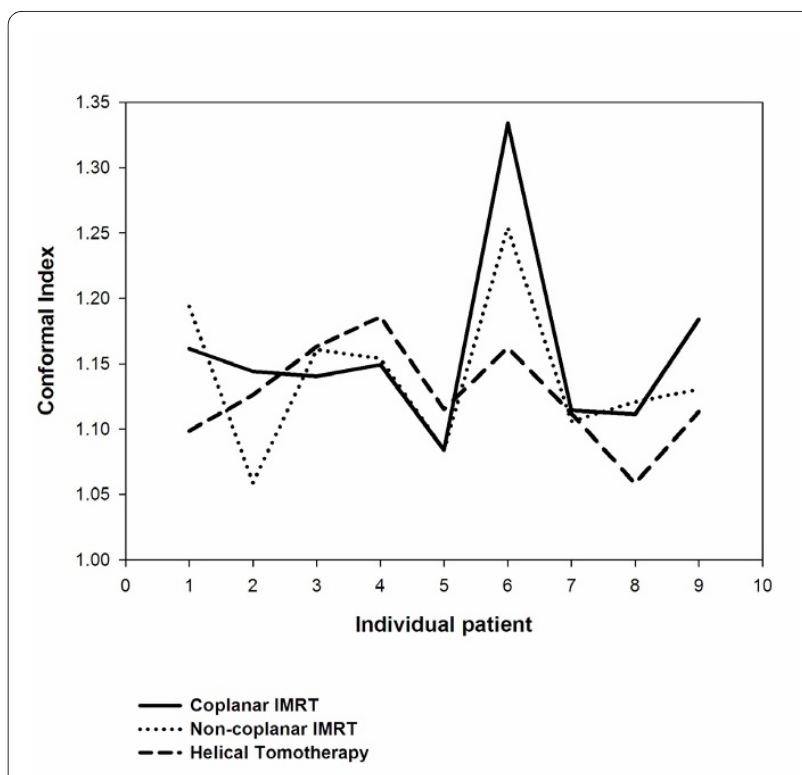

Figure 4 The conformal index $(\mathrm{Cl})$ for each individual patient undergoing coplanar intensity-modulated radiotherapy (IMRT), noncoplanar IMRT, and helical tomotherapy.

mean liver dose was less than $31 \mathrm{~Gy}\left(\mathrm{BED}=30 \mathrm{~Gy}_{10}\right.$ in 2 Gy/fraction) [28]. A mean dose to normal liver smaller than 23 Gy (4-6 Gy/fraction) or 30 Gy (2 Gy/fraction) could be safe parameters for RILD prevention as reported by Liang et al. [40] and Kim et al. [29], respectively (Table 2). The MTD model is based on PTV and liver volume in equal fractions. The concepts of dose constraints for normal organs are extrapolated from the critical volume model [41] as well as the known constraints on partial liver resection that have indicated that up to $80 \%$ of the liver can be safely removed in a patient with adequate liver function [42]. In addition, the constraint of $700 \mathrm{cc}$ or $35 \%$ of normal liver to receive less than $15 \mathrm{~Gy}$, as no significant instances of RILD have been reported [43].

Additionally, Yamada et al. [44] reported that deterioration of liver function was observed in all patients with $\mathrm{V} 30>40 \%$. Chen JC et al. suggested that V30 < 42\% could avoid RILD [45]. (Table 2) In current study, coplanar IMRT, noncoplanar IMRT and HT provide V30 data as $21 \%, 17 \%$ and $17 \%$, respectively. In the other words, noncoplanar IMRT and HT could be considered as another potential choice for HCC patients with PVT as compared with coplanar IMRT because they can achieve similar dose to the tumor with comparable UI and CI but a relatively low mean dose and V30 to the normal liver. Liang et al. [40] reported the tolerable liver volume percentages with 3DCRT planning with hypofractionation (4-6 Gy) was $35 \%$ for V25 (= V29 $\left.\mathrm{Gy}_{10}\right)$ and $28 \%$ for V30 (= V35 $\left.\mathrm{Gy}_{10}\right)$. (Table 2) In current study, coplanar IMRT provides similar results for the V10, V20 and V30 of normal liver as 3DCRT planning as compared to the previous report 
Table 1: Comparison of dosimetric parameters for irradiation of portal vein thrombosis and target volumes and normal organs at risk (OARs) by using different treatment techniques.

\begin{tabular}{|c|c|c|c|c|}
\hline & & Coplanar IMRT & Noncoplanar IMRT & Tomotherapy \\
\hline CTV & V95\% (\%) & $98.72 \pm 1.90$ & $99.98 \pm 0.02$ & $99.97 \pm 0.07$ \\
\hline \multirow[t]{4}{*}{ PTV } & V90\% (\%) & $99.44 \pm 1.07$ & $99.54 \pm 0.61$ & $99.84 \pm 0.17$ \\
\hline & V95\% (\%) & $98.83 \pm 0.74$ & $98.71 \pm 1.28$ & $99.17 \pm 0.64$ \\
\hline & UI & $1.10 \pm 0.02$ & $1.11 \pm 0.03$ & $1.04 \pm 0.02^{\#, *}$ \\
\hline & $\mathrm{Cl}$ & $1.16 \pm 0.07$ & $1.14 \pm 0.06$ & $1.13 \pm 0.04$ \\
\hline \multirow[t]{4}{*}{ Normal liver } & V10 (\%) & $64.81 \pm 17.86$ & $51.91 \pm 21.56$ & $72.51 \pm 13.31^{*}$ \\
\hline & V20 (\%) & $41.36 \pm 13.99$ & $32.62 \pm 14.95$ & $32.78 \pm 9.18$ \\
\hline & V30 (\%) & $21.10 \pm 7.90$ & $17.17 \pm 8.85$ & $17.00 \pm 6.10$ \\
\hline & mean (Gy) & $18.23 \pm 3.11$ & $16.14 \pm 4.61$ & $17.93 \pm 2.83$ \\
\hline Stomach & mean (Gy) & $11.68 \pm 5.47$ & $9.90 \pm 6.18$ & $13.19 \pm 6.05$ \\
\hline Right Kidney & mean (Gy) & $5.07 \pm 4.99$ & $6.32 \pm 5.08$ & $9.00 \pm 8.94$ \\
\hline Left Kidney & mean (Gy) & $2.1 \pm 3.03$ & $2.36 \pm 2.91$ & $5.00 \pm 5.27$ \\
\hline Spinal cord & D1\% (Gy) & $20.98 \pm 7.51$ & $20.12 \pm 9.08$ & $22.53 \pm 3.31$ \\
\hline $\begin{array}{l}\text { The Vx is the } p \\
\text { V90 and V95 } \\
\text { Ul: Uniformity } \\
\text { \#: The } p \text { value } \\
\text { *: The } p \text { value }\end{array}$ & $\begin{array}{l}\text { I liver volum } \\
\text { d by } 90 \% \text { an } \\
\text { lindex. } \\
\text { ng tomothe } \\
\text { ng tomother }\end{array}$ & $\begin{array}{l}\geq \times \text { Gy in the tota } \\
\text { cribed dose, respec } \\
\text { anar IMRT. } \\
\text { coplanar IMRT. }\end{array}$ & liver volume. & \\
\hline
\end{tabular}

[40]. In the mean time, the noncoplanar IMRT and tomotherapy techniques reduced more than $10 \%$ for V20 and V30 of normal liver, respectively. (Table 1) Compared with IMRT, HT has an additional dose superior and inferior to the target volume (Fig. 1) due to the thicker fan beam thickness [46]. Although HT shows greater conformity in the axial view as the dose was delivered rotationally with higher intensity modulation can be achieved. However, HT had greater V10 than the other modalities noted in the current study. (Table 1) The potential risk of radiation toxicities caused by low dose off-targets even with highly conformal radiotherapy has been reported [30]. Careful considerations should be taken into account for the larger low-dose regions to avoid unexpected side effects. According to our results and the guidelines of reducing the potential risk of RILD, we suggest that the constraints for the liver in the V30 for coplanar IMRT $v s$. noncoplanar IMRT vs. tomotherapy could be reconsidered as $21 \%$ vs. $17 \%$ vs. $17 \%$, respectively. Using IMRT or $\mathrm{HT}$, the constraints for mean dose to the normal liver could be reconsidered as below: when delivering $50 \mathrm{~Gy}$ and 60-66 Gy to the tumor bed, the mean dose to the normal liver could be less than 20 Gy and 25 Gy, respectively.
The constraints for liver could be more tighten than those used in 3DCRT when we used IMRT or HT for HCC patients with PVT.

The rates of gastrointestinal complications linked to doses of < $40 \mathrm{~Gy}, 40-50 \mathrm{~Gy},>50$ Gy were 4.2\%, 9.9\%, and $13.2 \%$, respectively [16]. Both IMRT techniques and HT had similar dosimetric effects for OARs. Theoretically, these advantages allow these techniques to push the higher radiation dose to the tumor and keep relatively lower radiation dose to OARs (Table 1).

The applications for reducing liver motion are not used in the current study. To reduce the motion of liver in radiotherapy, several strategies have been reported. The application of four-dimensional computed tomography (4D CT) using an external respiratory signal to acquire difference phases of CT images could improve the dose coverage for target volumes [47]. Further use of abdominal compression was showed effectively in reducing liver tumor motion, yielding small and reproducible excursions in three dimensions [48]. Case et. al. [49] showed that the change in liver motion amplitude was minimal over the treatment course and no apparent relationships with the magnitude of liver motion and intrafraction 
Table 2: The parameters of predicted helical tomotherapy plan within mean $30 \mathrm{~Gy}$ to normal liver of hepatocellular carcinoma compared with selected published series.

\begin{tabular}{|c|c|c|c|c|}
\hline Published series & Modality & $\begin{array}{l}\text { Mean Tumor dose } \\
\text { (Gy) } \\
\text { /fraction size (Gy) }\end{array}$ & V30 (\%) & $\begin{array}{l}\text { Suggested mean dose (Gy) of } \\
\text { normal liver under } \\
\text { radiotherapy }\end{array}$ \\
\hline Dawson et al. [28] & 3DCRT & $52.5 / 1.5-1.65$ & & $30 \mathrm{~Gy}_{10}$ \\
\hline Kim et al. [29] & 3DCRT & $54 / 2$ & & $30 \mathrm{~Gy}$ \\
\hline Cheng et al. [45] & 3DCRT & $50 / 1.8-2$ & $42 \%$ & \\
\hline Liang et al. [40] & 3DCRT & $50 / 4-6$ & $35 \%$ & $28 \mathrm{~Gy}_{10}$ \\
\hline Yamada et al. [44] & 3DCRT & $57 / 2$ & $40 \%$ & \\
\hline IMRT & Coplanar IMRT & $50.4 / 1.8$ & $21 \%$ & $20 \mathrm{~Gy}$ \\
\hline IMRT & Noncoplanar IMRT & $50.4 / 1.8$ & $17 \%$ & $20 \mathrm{~Gy}$ \\
\hline HT & Tomotherapy & $50.4 / 1.8$ & $17 \%$ & $20 \mathrm{~Gy}$ \\
\hline
\end{tabular}

Abbreviations: $3 \mathrm{DCRT}=$ Three-dimensional conformal radiotherapy; $\mathrm{HT}=$ Helical tomotherapy; $\mathrm{V} 30=$ percent volume of normal liver with radiation dose more than $30 \mathrm{~Gy} ; X_{\mathrm{G} y_{10}}=$ a biologic effective dose (BED) of $X \mathrm{~Gy}_{10}$ as the $\alpha / \beta$ ratio $=10$ in daily fraction of $2 \mathrm{~Gy}$; OARs $=$ Organs at risk.

time. The application of 4D CT and abdominal compression may thus increase the coverage of target volume and reduce the motion uncertainty in radiation therapy.

\section{Conclusions}

To sum up, our results suggest that noncoplanar IMRT and HT are potentially effective techniques of radiation therapy for HCC patients with PVT. Constraints for the liver in IMRT and HT could be stricter than for 3DCRT. Further clinical studies of HT and noncoplanar IMRT applied to HCC patients with PVT are warranted.

\section{Competing interests}

We have no personal or financial conflict of interest and have not entered into any agreement that could interfere with our access to the data on the research, or upon our ability to analyze the data independently, to prepare manuscripts, and to publish them.

\section{Authors' contributions}

All authors read and approved the final manuscript. CHH, CYL and PWS carried out all CT evaluations, study design, target delineations and interpretation of the study. CHH drafted the manuscript. CJ C, CCL, TEW, SCL, MJC and KHC took care of patients. HCT, NSC and HJT made the treatment planning and carried out all plans comparisons and evaluations. DYCH and YJ C participated in manuscript preparation and study design. LY W and YPH gave advice on the work and carried out statistical analysis.

\section{Author Details}

'Department of Radiation Oncology, Far Eastern Memorial Hospital, Taipei, Taiwan, 2Department of Surgery, Far Eastern Memorial Hospital, Taipei, Taiwan, ${ }^{3}$ Department of Radiation Oncology, Mackay Memorial Hospital, Taipei, Taiwan , ${ }^{4}$ Department of Gastrointestinal Division, Mackay Memorial Hospital, Taipei, Taiwan, ${ }^{5}$ Department of Medical Research, Mackay Memorial Hospital, Taipei, Taiwan, ${ }^{6}$ Institute of Traditional Medicine, School of Medicine, National YangMing University, Taipei, Taiwan, ${ }^{7}$ Department of Radiation Oncology, National Defense Medical Center, Taipei, Taiwan, ${ }^{8}$ Graduate Institute of Sport Coaching Science, Chinese Culture University, Taipei, Taiwan, ${ }^{95}$ chool and Graduate Institute of Physical Therapy, College of Medicine, National Taiwan University, Taipei, Taiwan, ${ }^{10}$ Department of Healthcare Administration, Asia University, Taichung, Taiwan and ${ }^{11}$ Department of Medical Physics, Memorial SloanKettering Cancer Center, New York, NY, USA
Received: 2 February 2010 Accepted: 23 May 2010

Published: 23 May 2010

References

1. Cook GC, B M: Hepatocellular carcinoma: One of the world's most common malignancies. Am J Med 1985, 233:705-08.

2. Parkin DM: Global cancer statistics in the year 2000. Lancet Oncol 2001 , 2:533-43.

3. Jemal A, Siegel R, Ward E, Hao Y, Xu J, Thun MJ: Cancer statistics, 2009. CA Cancer J Clin 2009, 59:225-49.

4. Albacete RA, Matthews MJ, Saini N: Portal vein thromboses in malignant hepatoma. Ann Intern Med 1967, 67:337-48.

5. Price J, Chan M, Hamilton-Wood C, Chronos NA, Mok SD, Metreweli C: Sonographic diagnosis of portal vein invasion in patients with hepatocellular carcinoma: comparison with arterial portography. Clin Radiol 1990, 41:9-12.

6. Stuart KE, Anand AJ, Jenkins RL: Hepatocellular carcinoma in the United States. Prognostic features, treatment outcome, and survival. Cancer 1996, 77:2217-22

7. Ceccotti P, Leen E, Kalogeropoulou CP, Visco-Comandini U, Sartori S, Caratozzolo M: Portal vein thrombosis may alter the correct evaluation of hepatocellular carcinoma with the sonographic contrast pulse sequence technique. J Ultrasound Med 2006, 25:1619-23.

8. Okuda K, Ohtsuki T, Obata H, Tomimatsu M, Okazaki N, Hasegawa H, Nakajima Y, Ohnishi K: Natural history of hepatocellular carcinoma and prognosis in relation to treatment. Study of 850 patients. Cancer 1985, 56:918-28.

9. Urata K, Matsumata T, Kamakura T, Hasuo K, Sugimachi K: Lipiodolization for unresectable hepatocellular carcinoma: an analysis of 205 patients using univariate and multivariate analysis. J Surg Oncol 1994, 56:54-8.

10. Fujii T, Takayasu K, Muramatsu Y, Moriyama N, Wakao F, Kosuge T, Takayama T, Makuuchi M, Yamasaki S, Okazaki N, et al:: Hepatocellular carcinoma with portal tumor thrombus: analysis of factors determining prognosis. Jpn J Clin Oncol 1993, 23:105-9.

11. Kumada K, Ozawa K, Okamoto R, Takayasu T, Yamaguchi M, Yamamoto Y, Higashiyama H, Morikawa S, Sasaki H, Shimahara Y, et al.: Hepatic resection for advanced hepatocellular carcinoma with removal of portal vein tumor thrombi. Surgery 1990, 108:821-7.

12. Yen FS, Wu JC, Kuo BI, Chiang JH, Chen TZ, Lee SD: Transcatheter arterial embolization for hepatocellular carcinoma with portal vein thrombosis. J Gastroenterol Hepatol 1995, 10:237-40.

13. Sakon M, Nagano H, Dono K, Nakamori S, Umeshita K, Yamada A, Kawata S, Imai Y, lijima S, Monden M: Combined intraarterial 5-fluorouracil and subcutaneous interferon-alpha therapy for advanced hepatocellular 
carcinoma with tumor thrombi in the major portal branches. Cancer 2002, 94:435-42.

14. Ohto M, Yoshikawa M, Saisho H, Ebara M, Sugiura N: Nonsurgical treatment of hepatocellular carcinoma in cirrhotic patients. World J Surg 1995, 19:42-6.

15. Stillwagon GB, Order SE, Guse C, Klein JL, Leichner PK, Leibel SA, Fishman EK: 194 hepatocellular cancers treated by radiation and chemotherapy combinations: toxicity and response: a Radiation Therapy Oncology Group Study. Int J Radiat Oncol Biol Phys 1989, 17:1223-9.

16. Park HC, Seong J, Han KH, Chon CY, Moon YM, Suh CO: Dose-response relationship in local radiotherapy for hepatocellular carcinoma. Int J Radiat Oncol Biol Phys 2002, 54:150-5.

17. Kim DY, Park W, Lim DH, Lee JH, Yoo BC, Paik SW, Kho KC, Kim TH, Ahn YC, Huh SJ: Three-dimensional conformal radiotherapy for portal vein thrombosis of hepatocellular carcinoma. Cancer 2005, 103:2419-26.

18. Toya R, Murakami R, Baba Y, Nishimura R, Morishita S, Ikeda O, Kawanaka K, Beppu T, Sugiyama S, Sakamoto T, Yamashita Y, Oya N: Conformal radiation therapy for portal vein tumor thrombosis of hepatocellular carcinoma. Radiother Oncol 2007, 84:266-71.

19. Hsieh CH, Jeng KS, Lin CC, Chen CK, Liu CY, Lin CP, Tai HC, Wang CH, Shueng PW, Chen YJ: Combination of sorafenib and intensity modulated radiotherapy for unresectable hepatocellular carcinoma. Clin Drug Investig 2009, 29:65-71.

20. Huang YJ, Hsu HC, Wang CY, Wang CJ, Chen HC, Huang EY, Fang FM, Lu SN: The Treatment Responses and Cases of Radiation Therapy to Portal Vein Thrombosis in Advanced Hepatocellular Carcinoma. Int J Radiat Oncol Biol Phys 2009, 73:1 155-63.

21. Verhey LJ: Comparison of three-dimensional conformal radiation therapy and intensity-modulated radiation therapy systems. Semin Radiat Oncol 1999, 9:78-98.

22. Tai HC, Hsieh CH, Chao KS, Liu SH, Leu YS, Chang YF, Hsiao HT, Chang YC, Huang DY, Chen YJ: Comparison of radiotherapy strategies for locally advanced hypopharyngeal cancer after resection and ileocolic flap reconstruction. Acta Otolaryngol 2009, 129:311-7.

23. Mackie TR, Balog J, Ruchala K, Shepard D, Aldridge S, Fitchard E, Reckwerdt P, Olivera G, McNutt T, Mehta M: Tomotherapy. Semin Radiat Oncol 1999, 9:108-17.

24. Shueng PW, Lin SC, Chong NS, Lee HY, Tien HJ, Wu LJ, Chen CA, Lee JJ, Hsieh CH: Total marrow irradiation with helical tomotherapy for bone marrow transplantation of multiple myeloma: first experience in Asia. Technol Cancer Res Treat 2009, 8:29-38.

25. Cheng JC, Wu JK, Huang CM, Liu HS, Huang DY, Tsai SY, Cheng SH, Jian JJ, Huang AT: Dosimetric analysis and comparison of three-dimensional conformal radiotherapy and intensity-modulated radiation therapy for patients with hepatocellular carcinoma and radiation-induced liver disease. Int J Radiat Oncol Biol Phys 2003, 56:229-34.

26. Cheng JC, Chuang VP, Cheng SH, Huang AT, Lin YM, Cheng TI, Yang PS, You DL, Jian JJ, Tsai SY, Sung JL, Horng CF: Local radiotherapy with or without transcatheter arterial chemoembolization for patients with unresectable hepatocellular carcinoma. Int J Radiat Oncol Biol Phys 2000, 47:435-42.

27. Emami B, Lyman J, Brown A, Coia L, Goitein M, Munzenrider JE, Shank B, Solin $\sqcup$, Wesson M: Tolerance of normal tissue to therapeutic irradiation. Int J Radiat Oncol Biol Phys 1991, 21:109-22.

28. Dawson LA, Normolle D, Balter JM, McGinn CJ, Lawrence TS, Haken Ten RK: Analysis of radiation-induced liver disease using the Lyman NTCP model. Int J Radiat Oncol Biol Phys 2002, 53:810-21.

29. Kim TH, Kim DY, Park JW, Kim SH, Choi Jl, Kim HB, Lee WJ, Park SJ, Hong EK, Kim CM: Dose-volumetric parameters predicting radiation-induced hepatic toxicity in unresectable hepatocellular carcinoma patients treated with three-dimensional conformal radiotherapy. Int J Radiat Oncol Biol Phys 2007, 67:225-31.

30. Shueng PW, Lin SC, Chang HT, Chong NS, Chen YJ, Wang LY, Hsieh YP, Hsieh CH: Toxicity risk of non-target organs at risk receiving low-dose radiation: case report. Radiat Oncol 2009, 4:71.

31. ICRU: Prescribing, recording and reporting photon beam therapy (supplement to ICRU Report 50). International Commission of Radiation Units and Measurements: ICRU 62. Bethesda, MD; 1999.

32. Sheng K, Molloy JA, Read PW: Intensity-modulated radiation therapy (IMRT) dosimetry of the head and neck: a comparison of treatment plans using linear accelerator-based IMRT and helical tomotherapy. Int J Radiat Oncol Biol Phys 2006, 65:917-23.
33. Mackie TR: History of tomotherapy. Phys Med Biol 2006, 51:R427-53.

34. Paddick I: A simple scoring ratio to index the conformity of radiosurgical treatment plans. Technical note. J Neurosurg 2000, 93(Suppl 3):219-22.

35. Wang X, Zhang X, Dong L, Liu H, Gillin M, Ahamad A, Ang K, Mohan R: Effectiveness of noncoplanar IMRT planning using a parallelized multiresolution beam angle optimization method for paranasal sinus carcinoma. Int J Radiat Oncol Biol Phys 2005, 63:594-601.

36. Ingold JA, Reed GB, Kaplan HS, Bagshaw MA: Radiation Hepatitis. Am J Roentgenol Radium Ther Nucl Med 1965, 93:200-8.

37. Lewin K, Millis RR: Human radiation hepatitis. A morphologic study with emphasis on the late changes. Arch Pathol 1973, 96:21-6.

38. Seong J, Park HC, Han KH, Chon CY: Clinical results and prognostic factors in radiotherapy for unresectable hepatocellular carcinoma: retrospective study of 158 patients. Int J Radiat Oncol Biol Phys 2003, 55:329-36.

39. Lawrence TS, Robertson JM, Anscher MS, Jirtle RL, Ensminger WD, Fajardo LF: Hepatic toxicity resulting from cancer treatment. Int J Radiat Oncol Biol Phys 1995, 31:1237-48

40. Liang SX, Zhu XD, Xu ZY, Zhu J, Zhao JD, Lu HJ, Yang YL, Chen L, Wang AY, Fu XL, Jiang GL: Radiation-induced liver disease in three-dimensional conformal radiation therapy for primary liver carcinoma: the risk factors and hepatic radiation tolerance. Int J Radiat Oncol Biol Phys 2006, 65:426-34.

41. Yaes RJ, Kalend A: Local stem cell depletion model for radiation myelitis. Int J Radiat Oncol Biol Phys 1988, 14:1247-59.

42. Penna C, Nordlinger B: Surgery of liver metastases from colorectal cancer: new promises. Br Med Bull 2002, 64:127-40.

43. Schefter TE, Kavanagh BD, Timmerman RD, Cardenes HR, Baron A, Gaspar LE: A phase I trial of stereotactic body radiation therapy (SBRT) for liver metastases. Int J Radiat Oncol Biol Phys 2005, 62:1371-8.

44. Yamada K, Soejima T, Sugimoto K, Mayahara H, Izaki K, Sasaki R, Maruta T, Matsumoto S, Hirota S, Sugimura K: Pilot study of local radiotherapy for portal vein tumor thrombus in patients with unresectable hepatocellular carcinoma. Jpn J Clin Oncol 2001, 31:147-52.

45. Cheng JC, Wu JK, Huang CM, Huang DY, Cheng SH, Lin YM, Jian JJ, Yang PS, Chuang VP, Huang AT: Radiation-induced liver disease after radiotherapy for hepatocellular carcinoma: clinical manifestation and dosimetric description. Radiother Oncol 2002, 63:41-5.

46. Oliver M, Ansbacher W, Beckham WA: Comparing planning time, delivery time and plan quality for IMRT, RapidArc and Tomotherapy. J App/ Clin Med Phys 2009, 10:3068.

47. Underberg RW, Lagerwaard FJ, Cuijpers JP, Slotman BJ, van Sornsen de Koste JR, Senan S: Four-dimensional CT scans for treatment planning in stereotactic radiotherapy for stage I lung cancer. Int J Radiat Oncol Biol Phys 2004, 60:1283-90.

48. Wunderink W, Romero Mendez A, de Kruijf W, de Boer H, Levendag P Heijmen B: Reduction of respiratory liver tumor motion by abdominal compression in stereotactic body frame, analyzed by tracking fiducial markers implanted in liver. Int J Radiat Oncol Biol Phys 2008, 71:907-15.

49. Case RB, Moseley DJ, Sonke JJ, Eccles CL, Dinniwell RE, Kim J, Bezjak A, Milosevic M, Brock KK, Dawson LA: Interfraction and Intrafraction Changes in Amplitude of Breathing Motion in Stereotactic Liver Radiotherapy. Int J Radiat Oncol Biol Phys 2010 in press.

doi: $10.1186 / 1748-717 X-5-40$

Cite this article as: Hsieh et al., Comparison of coplanar and noncoplanar intensity-modulated radiation therapy and helical tomotherapy for hepatocellular carcinoma Radiation Oncology 2010, 5:40 